\title{
Diane Desrosiers-Bonin, Éliane Viennot, a cura di, Actualité de Jeanne Flore
}

\section{Filippo Fonio}

\section{Q OpenEdition}

1 Journals

\section{Edizione digitale}

URL: http://journals.openedition.org/studifrancesi/34507

DOI: $10.4000 /$ studifrancesi.34507

ISSN: 2427-5856

\section{Editore}

Rosenberg \& Sellier

\section{Edizione cartacea}

Data di pubblicazione: 1 novembre 2005

Paginazione: 401-402

ISSN: 0039-2944

\section{Notizia bibliografica digitale}

Filippo Fonio, «Diane Desrosiers-Bonin, Éliane Viennot, a cura di, Actualité de Jeanne Flore», Studi Francesi [Online], 146 (XLIX | II) | 2005, online dal 30 novembre 2015, consultato il 20 avril 2021. URL: http://journals.openedition.org/studifrancesi/34507; DOI: https://doi.org/10.4000/studifrancesi. 34507

Questo documento è stato generato automaticamente il 20 avril 2021.

\section{(c) (1)}

Studi Francesi è distribuita con Licenza Creative Commons Attribuzione - Non commerciale - Non opere derivate 4.0 Internazionale. 


\title{
Diane Desrosiers-Bonin, Éliane Viennot, a cura di, Actualité de Jeanne Flore
}

\author{
Filippo Fonio
}

\section{NOTIZIA}

DIANE DESROSIERS-BONIN, ÉLIANE VIENNOT, a cura di (avec la collaboration de RÈGINE REYNOLDSCORNELL), Actualité de Jeanne Flore, Paris, Honoré Champion («Études et essais sur la Renaissance», 55), 2004, pp. 323.

1 Il volume raccoglie diciassette interventi relativi a diverse questioni che l'enigmatica figura di Jeanne Flore e la sua opera, i Comptes amoureux, suscitano. Alcuni dei contributi qui presenti sono stati raccolti nel corso della giornata della Renaissance Society of America (Firenze, marzo 2000) dedicata appunto a Jeanne Flore, altri sono la riedizione di studi non ancora tradotti in francese, altri infine sono inediti. L'iniziativa vale come testimonianza del recente interesse sorto intorno alla raccolta di novelle, in seguito anche all'edizione critica dei Comptes amoureux a cura di Gabriel-André Pérouse (Paris-Lyon, Éditions du CNRS-Presses universitaires de Lyon, 1980), della quale è fra l'altro prevista una ristampa. I Comptes amoureux par Madame Jeanne Flore, editi a Lyon per i tipi di Denis de Harsy fra gli anni Trenta e Quaranta del XVI secolo, costituiscono il primo esempio in Francia di una raccolta di novelle con cornice, sul modello decameroniano. L'oblio al quale l'opera pare condannata già dagli ultimi decenni del Cinquecento, va inteso tanto nell'ottica delle modalità di ricezione della scrittura femminile - o comunque sentita come tale - in epoca rinascimentale, quanto alla luce del destino che spesso accomuna i minores. La questione è ulteriormente complicata, nel caso dei Comptes amoureux, dall'incertezza nella quale giacciono allo stato attuale i problemi legati all'authorship della raccolta. Si discute infatti se sia opera di un uomo o di una donna, di un singolo o di un gruppo, e da ciò dipende in parte la lettura che dei Comptes amoureux può essere proposta. In particolare, il dibattito coinvolge quanti 
ritengono si tratti di opera proto-femminista, quanti invece considerano la raccolta una sorta di presa di posizione fra ironico e polemica, sulla scia della Querelle des femmes (DIANE DESROSIERS-BONIN, La réception critique des Comptes amoureux de Jeanne Flore, pp. 11-25). Alcuni dei contributi cercano appunto di aggirare la questione dello statuto autoriale posta in questi termini, occupandosi piuttosto della struttura ideologica sottesa al testo. NANCY FRELICK, considerando il problema dell'authorship dal punto di vista dell'orizzonte di attesa, ritiene di poter individuare nei Comptes amoureux un'economia discorsiva maschile, siano essi opera di un uomo o una donna: Attribuer un sexe à Jeanne Flore?, pp. 239-250.

2 Altri interventi cercano di ricostruire aspetti inerenti il milieu in cui nasce la raccolta, per proporre una datazione che tenga conto dell'attività dei circoli umanistici e degli stampatori lionesi e per tentare, nell'ipotesi che si tratti di opera collettiva, di formulare ipotesi circa gli autori di alcuni dei contes: WILLIAM KEMP, Denys de Harsy et François Juste vers 1540: de La Pugnition de l'Amour contempné aux Comptes amoureux?, pp. 269-291; RÉGINE REYNOLDS-CORNELL, Que cache le manteau de Jeanne Flore?, pp. 251-267. CLAUDE LA CHARITÉ si occupa della scelta del genere, del titolo della raccolta e delle occorrenze del termine compte all'interno dell'opera, in riferimento ai campi semantici del dilettevole, del verisimile, del favoloso (Le problème du genre dans les Comptes amoureux de Jeanne Flore: l'ambivalence du terme 'compte', pp. 209-225).

3 Fra le ipotesi formulate, è quella che un fantasma dello statuto autoriale possa essere ricercato nel testo, nella fattispecie nel personaggio di Madame Cebille. Il silenzio al quale la donna è di continuo ridotta sarebbe così la dinamica che fa nascere le novelle $\mathrm{e}$ le tiene unite. Tutto si svolge in funzione di Cebille, secondo una lettura che individua nel semiotico un campo marginale e sovversivo rispetto al simbolico, sulla scorta delle tesi di Julia Kristeva (CAROLYN M. FAY, Qui était Jeanne Flore? Subversion et silence dans Les Contes amoureux par Madame Jeanne Flore, pp. 93-112). JEAN-PHILIPPE BEAULIEU mette invece in evidenza l'impressione di ambiguità che si ricava dalla lettura, dovuta al gioco di equilibri che si crea fra cornice e novelle, e fra le novelle stesse. In base a riscontri di intertestualità interna, lo studioso nota una non-corrispondenza fra enunciati morali ed elementi narrativi delle novelle, in particolare nella misura in cui l'idea di un'assoluta preminenza dell'amore appare in contrasto con quella della possibilità di una libera scelta da parte della donna amante (L'ambiguité didactique dans les Comptes amoureux de Jeanne Flore, pp. 27-38). Lo scopo didattico e le modalità del discorso deliberativo, che suggerirebbero di leggere ogni novella come un exemplum a carattere di esortazione o dissuasione, è analizzato da MARIE CLAUDE MALENFANT (Le discours délibératif dans les Comptes amoureux de Jeanne Flore: 'exempla' et visées persuasives, pp. 57-73).

4 Centrale è appunto la questione dell'intertestualità dei Comptes amoureux: COLETTE H. WINN, Les Comptes amoureux de Jeanne Flore: un texte-écho, pp. 39-55; MAGDA CAMPANINI CATANI, Les Contes amoureux de Jeanne Flore: notes pour une étude intertextuelle, pp. 127-143; FLOYD GRAY, Jeanne Flore et le désir érotique: féminisme ou fantasme masculin?, pp. 165-186; SERGIO CAPPELLO, Le corps dans les Comptes amoureux: Pyralius le Jaloux, pp. 187-208. I contributi di Colette Winn e Floyd Gray sono dedicati a problemi di assimilazione di codici culturali cortesi nella raccolta dei Comptes amoureux (nella stessa direzione va l'analisi di LAURA DOYLE GATES, Le 'vray amour' dans les Comptes amoureux de 
Jeanne Flore, pp. 113-126), mentre Sergio Cappello e Magda Campanini Catani si occupano più da vicino del problema delle fonti di alcuni episodi delle novelle.

5 La questione del linguaggio del corpo e dei sensi nell'opera di Jeanne Flore è tematica affrontata in alcuni dei contributi sopraccitati, e costituisce l'oggetto dell'intervento di MANUELA ÁLVAREZ JURADO, L'aventure corporelle dans les Comptes amoureux de Jeanne Flore, pp. 75-92. La rappresentazione del corpo nei Comptes amoureux può anche prestarsi a un tipo di lettura anti-femminista, sul tipo di quella proposta da EMMA TYLER (Les Contes amoureux de Jeanne Flore et les fictions du sujet féminin, pp. 145-164) o da CATHLEEN M. BAUSCHATZ (Jeanne Flore derechef. La parodie d'œuvres de femmes dans les Comptes amoureux, pp. 293-300), mentre MARCEL TÉTEL evidenzia come la messa in discussione dell'istituto del matrimonio mediante il ridicolizzare le relazioni coniugali sia un carattere assolutamente moderno dei Comptes amoureux (L'audace des Comptes amoureux, pp. 227-237). L'esauriente Bibliografia è curata da DIANE DESROSIERS-BONIN ed HÉLÉNE LUCUIX. Segue un indice dei nomi di persona. 